\title{
Closing forensic psychiatric hospitals in Italy: a new revolution begins?
}

\author{
Corrado Barbui and Benedetto Saraceno
}

\section{Summary}

On 30 May 2014 the Italian Parliament approved a new law regarding forensic psychiatric hospitals. Forensic psychiatric hospitals are facilities that admit individuals who have committed a criminal offence but lack criminal responsibility because of a mental disorder and are deemed as dangerous to public safety. Here we report the key aspects of the new legislation together with some critical considerations.

\section{Declaration of interest}

None.

\section{Copyright and usage}

(c) The Royal College of Psychiatrists 2015.
Corrado Barbui (pictured) coordinates the activities of the WHO Collaborating Centre for Research and Training in Mental Health and Service Evaluation at the University of Verona, Italy, and conducts research in the field of evidencebased clinical psychopharmacology and pharmacoepidemiology. Benedetto Saraceno is a psychiatrist who has made, and continues to make, a major contribution to global mental health, by his sustained and vigorous efforts for the advancement of psychiatry and mental health around the world, and particularly for those who are least able to speak for themselves.

\section{The current legislative framework}

In Italy, current procedures relating to the management of individuals with mental disorders who have committed a criminal offence are based on assessment of criminal responsibility and dangerousness. Criminal responsibility refers to the ascertainment of whether a person with mental disorder should be relieved of the consequences of criminal conduct. In the case of full criminal responsibility there is no special pathway and the person with mental disorder receives a sentence and standard mental healthcare, including mental healthcare in prison. In cases where the court takes the decision that there is a lack of criminal responsibility, there are two possibilities. If the person is considered not dangerous to public safety, they are set free and they receive standard mental healthcare; whereas if the person is judged to be dangerous to public safety, they are admitted to a forensic psychiatric hospital or to an alternative sheltered facility. Dangerousness to public safety is then regularly assessed and an individual's classification as dangerous can be renewed or removed by the courts. If the latter, the person goes back into the community and standard mental healthcare is provided by community mental health services. ${ }^{1}$

\section{Forensic psychiatric hospitals in Italy}

Currently in Italy there are six forensic psychiatric hospitals with a total population of around 1000 individuals. Unfortunately, there is little epidemiological data on this population and on the quality of mental healthcare provided. ${ }^{1}$ These facilities are obsolete, with heavy use of custodial staff. With the exception of one hospital, the quality of mental healthcare is seriously unsatisfactory, as pointed out by a warning of human rights violations issued by the Council of Europe in 2006. Another critical point is that in too many cases dangerousness to public safety, the key condition for not being discharged, is renewed and extended with no limit, on the basis of a lack of family and social conditions suitable for discharge, rather than on the basis of an assessment of individual factors, a phenomenon called 'white life sentence'.

\section{The new legislation}

Within this context, in April 2008 the Italian government issued a first decree that established a programme for progressive downsizing and closure of the six forensic psychiatric hospitals, transferring responsibilities and resources to the National Health System. In 2012, a new law (Law 9/2012) established that new residential facilities had to be developed to better meet the needs of providing intensive and high-quality mental healthcare to socially dangerous individuals with mental disorders under proper secure conditions. These small-scale facilities (no more than 20 individuals, up to 4 patients per bedroom) are intended to replace admissions to forensic psychiatric hospitals.

As initial implementation of these laws has been unsatisfactory, in May 2014 Law 81/2014 set deadlines and operational procedures that mandate regional authorities to strictly follow the following process. For individuals already admitted to forensic psychiatric hospitals, individualised discharge programmes must be developed within a given deadline; if dangerousness to public safety is renewed by the court, discharge programmes must assure secure conditions, which may include transition from a forensic psychiatric hospital to one of the newly established small-scale residential facilities or placement in the community under the adoption of less restrictive security measures.

For new cases, i.e. individuals with mental disorders who commit a crime and are deemed as dangerous to public safety, a similar pathway must be followed, including the possibility of admission to one of the newly established residential facilities. In exceptional circumstances, which must be documented, patients may still be admitted to, or not discharged from, a forensic psychiatric hospital, provided that there are no other therapeutic options that assure proper secure conditions. The deadline for final dismantlement of forensic psychiatric hospitals is the end of March 2015.

According to the new law, dangerousness to public safety can only be motivated by reasons related to the patient's individual characteristics, and can no longer be motivated by family, social and economic reasons, including the lack of an individualised mental healthcare programme. Additionally, the new law states that security measures can be renewed up to a maximum number of years that correspond to the sentence that would have been given if the individual had committed the same crime without 
having a mental disorder. Finally, the law appointed a national expert committee with monitoring responsibilities.

\section{Critical aspects of the new law}

As expected, the new law has activated a heated debate among Italian mental health professionals. As a general point it should be emphasised that this reform has been approved without clear cut evidence of its cost-effectiveness. Similarly, the results of studies describing the outcomes of patients discharged from forensic psychiatric hospitals are unavailable, and no recent and reliable information on the clinical characteristics and care needs of forensic psychiatric patients have been collected. ${ }^{2}$ However, lack of data in this setting is a general problem, as there is a surprising shortage of basic information and evidence about the quality and effectiveness of the various legal frameworks and forensic care provisions throughout the European Union member states. ${ }^{3}$

More specifically, the following criticisms have been raised. First, the law designed a double pathway, as it does not state that admission to forensic psychiatric hospitals is forbidden, although it explicitly states it should be exceptional. This may lead to a double standard of mental healthcare (forensic psychiatric hospital v. small-scale residential facility) with potential inequalities in the provision of therapeutic programmes and economic implications that may not be sustainable. A second issue is that with the new system assessment of criminal responsibility becomes a crucial factor; individuals who have committed a crime may attempt to demonstrate lack of criminal responsibility because this would lead to admission to a small-scale therapeutic facility rather than to prison. If this happens, individuals without a mental disorder may use the resources allocated to individuals with mental disorders with no expected beneficial consequences. This may ultimately distort the mission of mental healthcare towards a requirement for control and security rather than care. Third, another critical consideration is the extra burden that community services will face. Several facilities in Italy are presently understaffed and in the past few years economic resources have been cut, to a varying degree, across the country. Additional resources will also be needed to increase the competence of mental health professionals working in community services in treating criminal offenders with mental disorders.

It has been argued that the reforms should have been even more radical, for example by cancelling the legal concept of criminal responsibility. This would simplify the whole system, as individuals with mental disorders who commit a crime would receive the same sentence as those who do not have a mental disorder, with the addition of appropriate mental healthcare. We argue that this would represent a practical way for completely destigmatising individuals with mental disorders, who would eventually be managed in the same way as all other individuals.
Finally, it has been highlighted that the new law, by allowing the development of new psychiatric facilities, may eventually produce a transition of individuals from large forensic psychiatric hospitals to small-scale psychiatric hospitals, with a final outcome of trans-institutionalisation. In some cases, these new facilities have already been planned to be placed in the sites of the 'old' psychiatric hospitals, closed in Italy more than 35 years ago by Law 180. ${ }^{4}$ This has been seen as a 're-opening' of psychiatric institutions, with no real advantage for patients and with an evident trend to step back from the innovations of Law 180 .

\section{A new revolution begins?}

After more than 35 years since the passing of Law 180, the reform law that marked the phasing out of psychiatric hospitals and the gradual development of a community-based system of psychiatric care, ${ }^{4}$ we hope that the revision and implementation of Law $81 / 2014$ may progressively increase the quality of mental health care provided to individuals with mental disorders who committed a crime, paying due respect to human rights issues. ${ }^{5}$ Considering that the number of individuals affected by this law is relatively small, we argue that a national epidemiological registry might be developed to closely monitor the whole implementation process, aiming for continuous improvement.

Corrado Barbui, MD, WHO Collaborating Centre for Research and Training in Menta Health and Service Evaluation, Department of Public Health and Community Medicine Section of Psychiatry, University of Verona, Verona, Italy; Benedetto Saraceno, MD, Gulbenkian Global Mental Health Platform, Lisbon, Portugal.

Correspondence: Corrado Barbui, MD, Section of Psychiatry, Policlinico G.B. Rossi, Piazzale Scuro 10, 37134 Verona, Italy. Email: corrado.barbui@univr.it

First received 30 Jun 2014, final revision 6 Nov 2014, accepted 2 Dec 2014

\section{References}

1 Peloso PF, D'Alema M, Fioritti A. Mental health care in prisons and the issue of forensic hospitals in Italy. J Nerv Ment Dis 2014; 202: 473-8.

2 Castelletti L, Lasalvia A, Molinari E, Thomas SDM, Straticò E, Bonetto C. A standardised tool for assessing needs in forensic psychiatric population: clinical validation of the Italian CANFOR, staff version. Epidem Psych Sci 2014; Oct 13 (Epub ahead of print).

3 Salize HJ, Lepping P, Dressing H. How harmonized are we? Forensic mental health legislation and service provision in the European Union. Crim Behav Mental Health 2005; 15: 143-7.

4 Barbui C, Tansella M. Thirtieth birthday of the Italian psychiatric reform: research for identifying its active ingredients is urgently needed. J Epidemiol Community Health 2008; 62: 1021.

5 Drew N, Funk M, Tang S, Lamichhane J, Chávez E, Katontoka S, et al. Human rights violations of people with mental and psychosocial disabilities: an unresolved global crisis. Lancet 2011; 378: 1664-75. 